\section{Antibodies: from genes to targeted cancer gene therapy}

Since the development of monoclonal antibodies by Köhler and Milstein in 1975 they have been extensively explored as therapeutic agents for a wide range of diseases. There have been some successes in the treatment of malignant disease; notably for B cell lymphoma, for the adjuvant therapy of colorectal cancer and, combined with chemotherapy, in the treatment of metastatic breast cancer. The role of antibodies as protein therapeutics continues to expand but they also provide a flexible means of targeting which could have broad application to targeted cancer gene therapy. Developments in antibody technology allow the production of novel fragments from pre-existing hybridomas that have many potential uses. In addition, the phage antibody system provides the ability to produce such fragments de novo. ${ }^{1}$ Recently, it has been established that these single chain $\mathrm{Fv}(\mathrm{scFv})$ fragments function well in tumour imaging studies. ${ }^{2}$ Such scFvs, principally because they are made as genetic fragments and can be expressed from a single cassette, are ideally suited for many gene therapy applications (Figure 1). In recent issues of Gene Therapy and other journals there have been a number of papers describing the

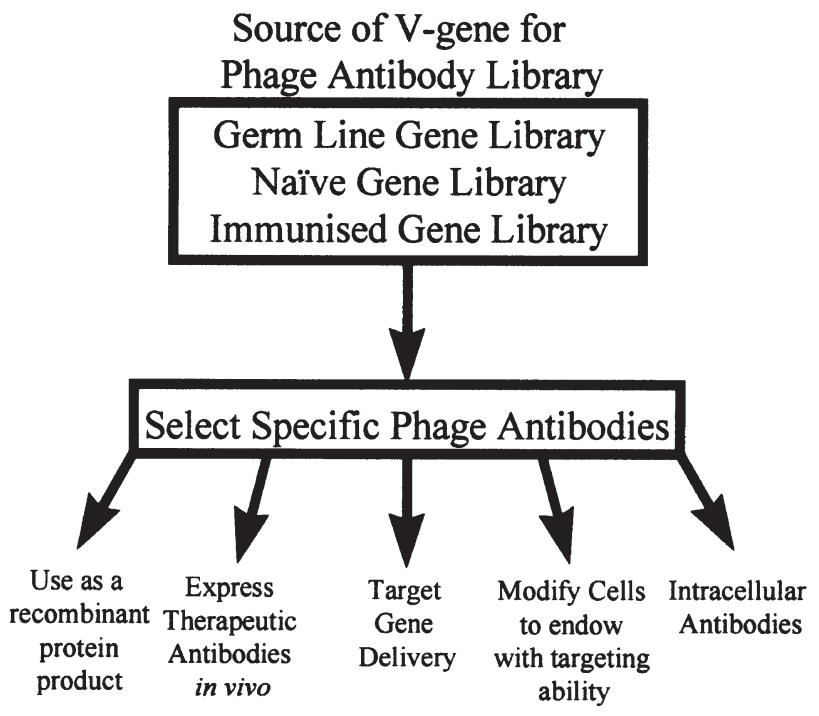

GENE THERAPY APPLICATIONS

Figure 1 Antibodies made from phage libraries as recombinant scFus are ideally suited to use in a range of gene therapy applications. application of such antibody fragments in each of these approaches. These are briefly discussed here.

\section{In vivo expression of antibodies}

Bacterial expression of recombinant antibody fragments was first described in 1988, yet despite the potential advantages of size (smaller size gives better penetration of tissues/tumours) and flexibility (ease of manipulation to generate fusion proteins) they remain to be tested clinically in therapeutic trials. This, in part, reflects the difficulty in producing large quantities of protein and of providing multiple doses/continuous infusions required to maintain appropriate circulating levels of small molecules. As a potential means of overcoming these difficulties, the in vivo expression of $\mathrm{scFv}$-fusion proteins using direct injection of recombinant adenovirus has been reported. ${ }^{3}$ Model experiments have shown that it is possible to obtain sustained levels of expression in mice at viral doses that are potentially achievable in patients. Adenovirus, however, has some limitations and if longer periods of production are necessary modifications to this system will be required. For example, it may be possible to use other serotypes of adenovirus to avoid the immune response to viral proteins. Alternatively, the use of encapsulated cells modified to express recombinant antibody fragments may be a feasible approach. ${ }^{4}$ However, unless autologous cells are used, there is a risk of immune destruction by antibody/complement even if the cells are protected from the cellular immune system. Whatever the final system used, these approaches should simplify the clinical testing of such recombinant antibody fragments as production will be facilitated. A further potential advantage is that the in vivo production results in an apparently more stable product with no detectable proteolysis of fusion products. ${ }^{3}$ The ability to produce the antibody fragments at the site required (eg for regional tumour therapy) may also bring benefits in terms of better efficacy due to higher local levels of antibody coupled with lower systemic toxicity.

\section{Targeting gene delivery}

The use of antibody fragments to target retroviruses was made possible by the retroviral display of $\mathrm{scFv} .{ }^{5}$ Recently, it has been reported that antibodies made by phage display could target gene delivery albeit relatively inefficiently. ${ }^{6}$ Such antibody fragments have also been used to produce reagents for the targeting of adenoviruses. ${ }^{7}$ In addition to targeting, adenoviral entry via the EGF receptor also enhances the efficiency of gene delivery. This approach is attractive for use with novel therapeutic strategies such as VDEPT (virally directed enzyme activated 
prodrug therapy) or the introduction of wild-type p53 into tumours as it should improve the efficiency of the process and reduce any toxicity. For this 'adenobody' approach the 'off-rate' of the antibody from the adenovirus will be critical and may require specific engineering of the $\mathrm{scFv}$ through antibody phage display technology. ${ }^{1}$ However, it remains to be seen how well such indirect methods of targeting will function in vivo and an alternative is to modify the fibre directly. ${ }^{8}$ Although more difficult to make, directly modified viruses will be more stable and if the engineered adenoviruses generally maintain efficiency of infection (unlike retroviruses) then this will be a preferable approach. Important physiological issues, such as access from the blood stream to solid tumours for large particles including viral vectors, remains a problem limiting the potential of targeted viral therapy for cancer. At least in the short term, such therapy is likely to be limited to compartmental (eg intraperitoneal, intravascular) or ex vivo applications. For the latter, a particularly interesting system is the inverse targeting described with retroviral vectors that could be useful for transducing bone marrow as part of a therapeutic approach. ${ }^{9}$ Gene delivery can potentially be targeted to either stem cell or tumour cell populations depending on the ligand (or scFv) displayed on the retrovirus.

\section{Cell targeting}

Cells interact through receptor-ligand binding which then produces many important functional effects. In principle, by modifying the external domain of any receptor or ligand to incorporate an antibody fragment, the interaction can be redirected, while the functional downstream effects are retained. An attractive example of this approach is to modify lymphocyte receptors so that the cells are endowed with antitumour/antiviral specificity. The most widely modified receptor is the CD3 receptor. Such T cells with chimeric receptors are able to redirect effector function to new targets and cytotoxicity is also MHC independent (important as many tumour cells down-regulate expression of $\mathrm{MHC}$ class I). ${ }^{10}$ Chimeric CD3 receptors can also be active in other cells and the approach of modifying haemopoietic stem cells allows the modification of many different cell types. Two recent papers have shown that this approach could be an effective means of tumour treatment. ${ }^{11,12}$ However, both also point to the important role of cells other then T cells (eg NK cells, neutrophils or macrophages) in tumour rejection. This may be because they are intrinsically more effective or because efficient $\mathrm{T}$ cell activation requires multiple receptor binding events. Specifically, naïve $\mathrm{T}$ cells require costimulation via the interaction of the B7 family with CD28 for full activation and this, potentially critical feature is lacking in approaches explored to date. Modification of CD28 by incorporating a scFv moiety on to the CD28 molecule can provide antigen-specific costimulation and this should enhance the antitumour capacity of T cells. ${ }^{13}$ It will be interesting to see if such double modified cells will be more effective and thus the $\mathrm{T}$ cell component of tumour protection more apparent. There are other receptors that could be modified but there are practical limitations and it may prove difficult to modify efficiently more than two receptors.

\section{Intracellular antibodies}

It has also been shown that antibodies can function intracellularly. Binding to abnormal proteins associated with malignant transformation can reverse that effect and it was recently demonstrated that an intracellular scFv antiRas delivered using a recombinant adenovirus could produce sustained regression of local tumour. ${ }^{14}$ For successful clinical development the key will be efficient (systemic) gene delivery and as indicated above targeted viral vectors may be advantageous.

Overall, although the use of $\mathrm{scFv}$ antibodies and their derivatives in gene therapy is in its infancy, there is enormous potential, both indirectly, by targeting and enhancing gene delivery and, more directly, as a component of the therapeutic modality. Isolating antibody fragments from phage display libraries should directly produce reagents suited for this use and facilitate testing the strategies outlined above.

\section{RE Hawkins ${ }^{1}$, HA Whittington ${ }^{1,2}$, SJ Watkins ${ }^{1}$ and DE Gilham ${ }^{1}$ \\ ${ }^{1}$ Department of Medical Oncology, Christie CRC Research Centre, Paterson Institute for Cancer Research, Wilmslow Road, Manchester M20 4BX; and ${ }^{2}$ Current address: Department of Medicine, University of Bristol, Bristol, UK}

\section{References}

1 Winter G, Griffiths AD, Hawkins RE, Hoogenboom HR. Making antibodies by phage display technology. Annu Rev Immunol 1994; 12: 433-455.

2 Begent RHJ et al. Single-chain Fv antibody selected from a combinatorial library: clinical evidence of efficient tumour targeting. Nature Med 1996; 2: 979-984.

3 Whittington HA, Ashworth LJ, Hawkins RE. Recombinant adenoviral delivery for in vivo expression of scFv antibody fusion proteins. Gene Therapy 1998; 5: 770-777.

4 Pelegrin $\mathrm{M}$ et al. Systemic long-term delivery of antibodies in immunocompetent animals using cellulose sulphate capsules containing antibody-producing cells. Gene Therapy 1998; 5: 828-834.

5 Russell SJ, Hawkins RE, Winter G. Retroviral vectors displaying functional antibody fragments. Nucleic Acids Res 1993; 21 1081-1085.

6 Martin F et al. Retroviral vector targeting to melanoma cells by single-chain antibody incorporation in envelope. Hum Gene Ther 1998; 9: 737-746.

7 Watkins SJ, Mesyanzhinov VV, Kurochkina LP, Hawkins RE The adenobody approach to viral targeting: specific and enhanced adenoviral gene delivery. Gene Therapy 1997; 4: 1004-1012.

8 Wickham TJ, Roelvink PW, Brough DE, Kovesdi I. Adenovirus targeted to heparan-containing receptors increases its gene delivery efficiency to multiple cell types. Nat Biotechnol 1996; 14: 1570-1573.

9 Fielding AK et al. Inverse targeting of retroviral vectors: selective gene transfer in a mixed population of hematopoietic and nonhematopoietic cells. Blood 1998; 91: 1802-1809.

$10 \mathrm{Hwu} \mathrm{P}$ et al. In vivo antitumor activity of $\mathrm{T}$ cells redirected with chimeric antibody/T cell receptor genes. Cancer Res 1995; 55: 3369-3374.

11 Hege KM et al. Systemic T cell independent tumour immunity after transplantation of universal receptor-modified bone marrow into SCID mice. J Exp Med 1996; 184: 2261-2269. 
12 Wang G et al. A T cell independent antitumour response in mice with bone marrow cells retrovirally transduced with an antibody/Fc- $\gamma$ chain chimeric receptor gene recognising a human ovarian cancer antigen. Nature Med 1998; 4: 168-172.

13 Alvarez-Vallina L, Hawkins RE. Antigen specific targeting of
CD28-mediated T cell costimulation using chimeric scFv-CD28 receptors. Eur J Immunol 1996; 26: 2304-2309.

14 Cochet $\mathrm{O}$ et al. Intracellular expression of an antibody fragmentneutralising p21 ras promotes tumor regression. Cancer Res 1998; 58: $1170-1176$. 